\title{
The Impact of Mitochondrial Dysfunction on Dopaminergic Neurons in the Olfactory Bulb and Odor Detection
}

\author{
Thomas $\mathrm{Pa}^{1}{ }^{1}$ (D) $\cdot$ Marlene Aßfalg ${ }^{1} \cdot$ Marianna Tolve $^{2} \cdot$ Sandra Blaess $^{2} \cdot$ Markus Rothermel $^{3} \cdot$ Rudolf J. Wiesner $^{1,4}$. \\ Konrad M. Ricke ${ }^{1,5}$
}

Received: 12 August 2019 / Accepted: 13 May 2020

(C) The Author(s) 2020

\begin{abstract}
Understanding non-motor symptoms of Parkinson's disease is important in order to unravel the underlying molecular mechanisms of the disease. Olfactory dysfunction is an early stage, non-motor symptom which occurs in 95\% of Parkinson's disease patients. Mitochondrial dysfunction is a key feature in Parkinson's disease and importantly contributes to the selective loss of dopaminergic neurons the substantia nigra pars compacta. The olfactory bulb, the first olfactory processing station, also contains dopaminergic neurons, which modulate odor information and thereby enable odor detection as well as odor discrimination. MitoPark mice are a genetic model for Parkinson's disease with severe mitochondrial dysfunction, reproducing the differential vulnerability of dopaminergic neurons in the midbrain. These animals were used to investigate the impact of mitochondrial dysfunction on olfactory-related behavior and olfactory bulb dopaminergic neuron survival. Odor detection was severely impaired in MitoPark mice. Interestingly, only the small anaxonic dopaminergic subpopulation, which is continuously replenished by neurogenesis, was moderately reduced in number, much less compared with dopaminergic neurons in the midbrain. As a potential compensatory response, an enhanced mobilization of progenitor cells was found in the subventricular zone. These results reveal a high robustness of dopaminergic neurons located in the olfactory bulb towards mitochondrial impairment, in striking contrast to their midbrain counterparts.
\end{abstract}

Keywords Parkinson's disease $\cdot$ Mitochondrial dysfunction $\cdot$ Olfactory bulb $\cdot$ Neurogenesis

\section{Introduction}

Parkinson's disease (PD) is the most common movement disorder affecting $1 \%$ of the population above 60 years [1] characterized by the initial loss of midbrain dopaminergic neurons (DaNs) in the substantia nigra pars compacta $(\mathrm{SNc})$. The

Electronic supplementary material The online version of this article (https://doi.org/10.1007/s12035-020-01947-w ) contains supplementary material, which is available to authorized users.

Thomas $\mathrm{Pa} \beta$

thomas.pass@uk-koeln.de

$\triangle$ Konrad M. Ricke

kricke@uottawa.ca

1 Center for Physiology and Pathophysiology, Institute of Vegetative Physiology, University of Cologne, Cologne, Germany

2 Neurodevelopmental Genetics, Institute of Reconstructive Neurobiology, University of Bonn School of Medicine \& University Hospital Bonn, Bonn, Germany depletion of striatal dopamine leads to progressive and irreversible motor impairment [2]. Besides the cardinal symptoms of $\mathrm{PD}$, non-motor dysfunctions including depression, sleep disturbance, and hyposmia have been described. Olfactory dysfunction is found in more than 95\% of PD patients and can precede motor symptoms by up to 10 years [3-5].

3 Institute for Biology II, Dept. Chemosensation, AG Neuromodulation, RWTH Aachen University, Aachen, Germany

4 Cologne Excellence Cluster on Cellular Stress Responses in Aging-associated Diseases (CECAD), University of Cologne, Cologne, Germany

5 Present address: Ottawa Hospital Research Institute, Brain and Mind Institute, University of Ottawa, 501 Smyth Rd., Ottawa, ON K1H8M5, Canada 
The olfactory bulb $(\mathrm{OB})$ is the first station of sensory processing in the olfactory system. Besides the midbrain, DaNs are also present in the $\mathrm{OB}$ and make up $5 \%$ of the neuronal population [6]. They function as interneurons and are necessary for odor discrimination rather than for odor detection $[7$, 8]. They are primarily located in the glomerular layer (GL), where they modulate the activity of both olfactory sensory fibers $[9,10]$ and mitral cells by $\mathrm{D}_{2}$ receptor-mediated inhibition $[11,12]$ as well as via contacts with external tufted cells [13]. Despite of multiple as well as contradictory statements regarding their categorization, OB DaNs can be clearly divided into small $(5-10 \mu \mathrm{m})$, anaxonic (SCs) and large (10-15 $\mu \mathrm{m}$ diameter), axonic cells (LACs) [14]. In contrast to LACs, SCs can be continuously generated throughout life by neurogenesis in mice as well as in humans [15-19]. Progenitor cells are formed in the dorsolateral region of the subventricular zone (SVZ) [20] and are characterized by the expression of the transcription factor PAX6, required for development into the $\mathrm{DaN}$ phenotype [21-23]. The majority of progenitor cells in the $\mathrm{OB}$ differentiate into interneurons in the granule cell layer, while only $5 \%$ of the $20,000-30,000$ newborn cells generated daily migrate to the GL $[24,25]$.

In PD, there are contradictory results according to the fate of DaNs in the OB. Luquin and colleagues reported elevated numbers of periglomerular DaNs, potentially displaying a compensatory mechanism induced by the early degeneration of other neurotransmitter systems and resulting in the olfactory dysfunction of patients [26]. In contrast, no difference in OB DaN numbers were found between PD patients and healthy individuals, implying that PD-related hyposmia is not due to alterations in the quantity of OB DaNs [27, 28].

Mitochondrial dysfunction is a central feature of PD, both in the common idiopathic as well as in the rare familiar forms caused by mutations, e.g. in the Parkin, Pink1, LRRK2, or DJ1 genes. During normal aging, SNc DaNs accumulate high loads of deletions in mitochondrial DNA (mtDNA), present in thousands of copies in neurons. Interestingly, this is accompanied by an upregulation of mtDNA copy numbers in healthy humans, while this compensatory mechanism is disrupted in PD patients [29]. This indicates that a defective mtDNA maintenance system and subsequent severe mitochondrial impairment is an important factor for the degeneration of SNc DaNs in PD.

The mitochondrial transcription factor A (TFAM) is crucial for mtDNA transcription and maintenance [30, 31]. Depletion of TFAM consequently leads to the loss of mtDNA encoded transcripts followed by a respiratory chain defect. MitoPark mice are lacking TFAM exclusively in DaNs, which culminates in progressive neuronal death and corresponding motor impairment starting from 14 weeks of age $[32,33]$. The progression of $\mathrm{PD}$ is recapitulated in terms of both anatomical and behavioral malfunctions. However, the impact of mitochondrial dysfunction on $\mathrm{OB}$ DaNs has not been investigated so far. Therefore, olfactory-related behavior was explored in MitoPark mice. Furthermore, mice were used to study the impact of mitochondrial defects on OB DaN survival as well as adult neurogenesis.

\section{Materials and Methods}

\section{Experimental Model}

All experiments were conducted in agreement with European and German guidelines and approved by local authorities (LANUV NRW; 81-02.04.2018-A210) (for breeding details, see supplementary methods). Experiments were carried out with male or female mice of the strain C57/BL6N. For the generation of MitoPark mice, DAT-cre mice (Cre-gene inserted upstream of the translation start codon in exon 2 of the DAT gene) and animals with a loxP-flanked Tfam allele were crossed as described in detail previously [32]. MitoPark mice (genotype Tfam $^{\text {loxPlloxP }}$, +/DAT-cre) show homozygous disruption of Tfam in dopaminergic neurons. Tfam ${ }^{\text {loxPlloxP }}$ and Dat-cre mice were provided by Nils-Göran Larsson (MaxPlanck-Institute for Biology of Ageing, Köln, Germany). $T_{\text {fam }}{ }^{\text {loxP/WT }}$ or Tfam ${ }^{\text {loxPlloxP }}$ mice were used as controls.

\section{COX-SDH Histochemistry}

Visualization of cytochrome c cxidase (COX) deficiency was performed by COX-SDH enzymatic activity staining [34]. COX is a respiratory chain (RC) complex, which is partially encoded by mtDNA, while succinate dehydrogenase (SDH), another respiratory chain enzyme, is entirely encoded by nuclear DNA. Impaired integrity of mtDNA results in COXdeficiency, but sustained SDH activity. Cells with decreased COX activity will stain blue, while cells with normal COX activity will appear brown (for details, see supplementary methods). Quantification of COX-deficient cells was performed at Bregma $+4.30 \mathrm{~mm}$ and $+3.00 \mathrm{~mm}$. Four images $(33 \times 50 \mu \mathrm{m})$ were taken from dorsal, ventral, and lateral OB regions per slice each. Number of COX-deficient cells was defined per slice.

\section{Immunohistochemistry}

Brain sections were stained for tyrosine hydroxylase (TH) to visualize DaNs and their projections (for details, see supplementary methods).

Soma size quantification of OB DaN subpopulations Soma sizes of DaNs stained for TH were analyzed by using FIJIsoftware (https://imagej.net/Fiji/Downloads). Cells were selected and automatically measured by the Wand-AutoTool based on the black-white contrast of the cells compared 
with background. The outline of the cell soma was detected, and the area within the shape was automatically calculated. Two subpopulations of DaNs with distinct morphological characteristics have been described [35]. DaN somata in the GL were differentiated into areas of $20-80 \mu \mathrm{m}^{2}$ for SCs and 80-180 $\mu^{2}$ for LACs. Two consecutive brain sections per mouse were analyzed at Bregma $+4.3,+3.75$, and +3.21 . Images were taken from both dorsal and ventral $\mathrm{OB}$ regions (for details, see supplementary methods).

\section{Analysis of Immunohistochemical Stainings}

Automated bright-field microscopy was done with a slide scanner (SCN400, Leica) equipped with a $40 \times$ objective. High-resolution images from the $\mathrm{OB}$ and striatum were generated by the Leica SlidePath Gateway and the Microsoft Image Composite Editor software. TH-positive striatal fiber density was determined by optical density (OD) analysis using FIJI-software (area fraction, https:// imagej.net/Fiji/Downloads) with two consecutive sections per mouse at Bregma $+0.74 \mathrm{~mm}$. Nonspecific background signal of the corpus callosum was subtracted from the striatal OD values. Fluorescence images were obtained by utilizing an inverse confocal microscope (TCS SP8 gSTED, Leica) with a $40 \times$ oil objective and the Leica Application Suite (LAS) 3 software. OB fluorescence microscopy was performed at Bregma $+4.30 \mathrm{~mm},+3.30 \mathrm{~mm}$, and $+2.30 \mathrm{~mm}$. TH- as well as PAX6-expressing cells were detected independently before merging the channels and quantifying the number of colocalizing cells, respectively. PAX6 expression in the SVZ was performed at Bregma +1 . $10 \mathrm{~mm}$, $+0.70 \mathrm{~mm}$, and $+0.30 \mathrm{~mm}$. Sections immunostained for EYFP/TH were imaged using with an inverted Zeiss AxioObserver Z1 microscope equipped with an ApoTome. Fluorescence images were acquired with Zeiss AxioCam MRm $1388 \times 1040$ pixels (Carl Zeiss). At 10× (EC PlnN 10x/0.3, Carl Zeiss), tile images were acquired with conventional epifluorescence. At 20× (EC PlnN 20x/ 0.5 , Carl Zeiss), tile images were acquired using the ApoTome function.

\section{Odor Discrimination Test}

Odor discrimination ability was examined to analyze DaN functionality in the OB. In addition, the odor discrimination test provides an opportunity to investigate odor detection rather independently from motor activity (for details of the test, see supplementary methods).

\section{Statistics}

Statistical analysis was done with GraphPad Prism 4 for Windows. Quantified data in the figures and in the text is presented as mean + SEM. Relative data is shown as percentage of control experiments. Values of sample size $(n)$ refer to mouse numbers. Unpaired $t$ tests, one-way or two-way ANOVA with post hoc comparisons (Bonferroni post hoc test) were used to determine differences between groups. A significance level of 0.05 was accepted for all statistical tests. Asterisks mark $P$ values of $0.05(*), 0.01(* *), 0.001(* * *)$, or 0.0001 (****).

\section{Results}

\section{Time Course of TFAM Loss in Different DaN Populations}

To determine the onset of Cre-mediated recombination, and thus TFAM knockout, in DaNs in the Dat ${ }^{\mathrm{Cre}}$ mouse line, we crossed $\mathrm{Dat}^{\mathrm{Cre} /+}$ mice with a reporter mouse line expressing enhanced yellow fluorescent protein (EYFP) (Rosa26 loxP-stop-loxP-EYFP $)$ [36]. In the midbrain, YFPexpression starts in laterally located TH-positive DaNs at E13.5 and spreads to medial DaN populations by E15.5 (Fig. 1) [37]. In the OB, we could not detect any YFPexpression in TH-positive DaNs at E15.5 (Fig. 1) or P0 (not shown), although some TH-positive neurons were present. Only at 4 weeks of age, TH-positive DaNs expressed YFP, indicating that recombination in $\mathrm{OB}$ DaNs only occurs postnatally in Dat ${ }^{\text {Cre }}$ mice. Consistent with this, RNA in situ hybridization data from the Allen Brain Atlas show that Dat is not expressed in the OB at P4 [38], while by P14, weak expression of DAT is detected in the $\mathrm{OB}$ and this is maintained into adulthood. These data suggest that inactivation of TFAM in the MitoPark model occurs at least 2 weeks later in OB DaNs than in midbrain DaNs.

\section{MitoPark Mice Show Dopaminergic Nigro-Striatal Degeneration}

TFAM depletion causes progressive motor impairment evoked by the loss of midbrain SNc DaNs and corresponding striatal fibers in the caudate putamen $(\mathrm{CPu})$ starting from 14 weeks of age $[32,33]$. Since Tfam inactivation in OB DaNs occurs later, 20- and 30-week-old MitoPark mice were used in this study. The severe degeneration of the nigrostriatal and the mesolimbic pathway was confirmed in 30week-old MitoPark mice. In particular, the reduction of THpositive cells in the SNc and ventral tegmental area (VTA) (Fig. 2a, b) as well as TH-staining in the corresponding striatal projection areas is dramatic (Fig. 2c, d). In addition, the olfactory tubercle (OT), located in the ventral striatum and innervated by VTA DaNs, shows lowered TH-staining. 
Fig. 1 Colocalization of TH and YFP is delayed in OB DaNs. In the midbrain, TH-positive cells are co-expressing YFP at both E15.5 and 4 weeks of age (yellow in merged panel). In contrast, colocalization of TH and YFP in the $\mathrm{OB}$ becomes apparent only at the age of 4 weeks. Scale bars: $100 \mu \mathrm{m}$

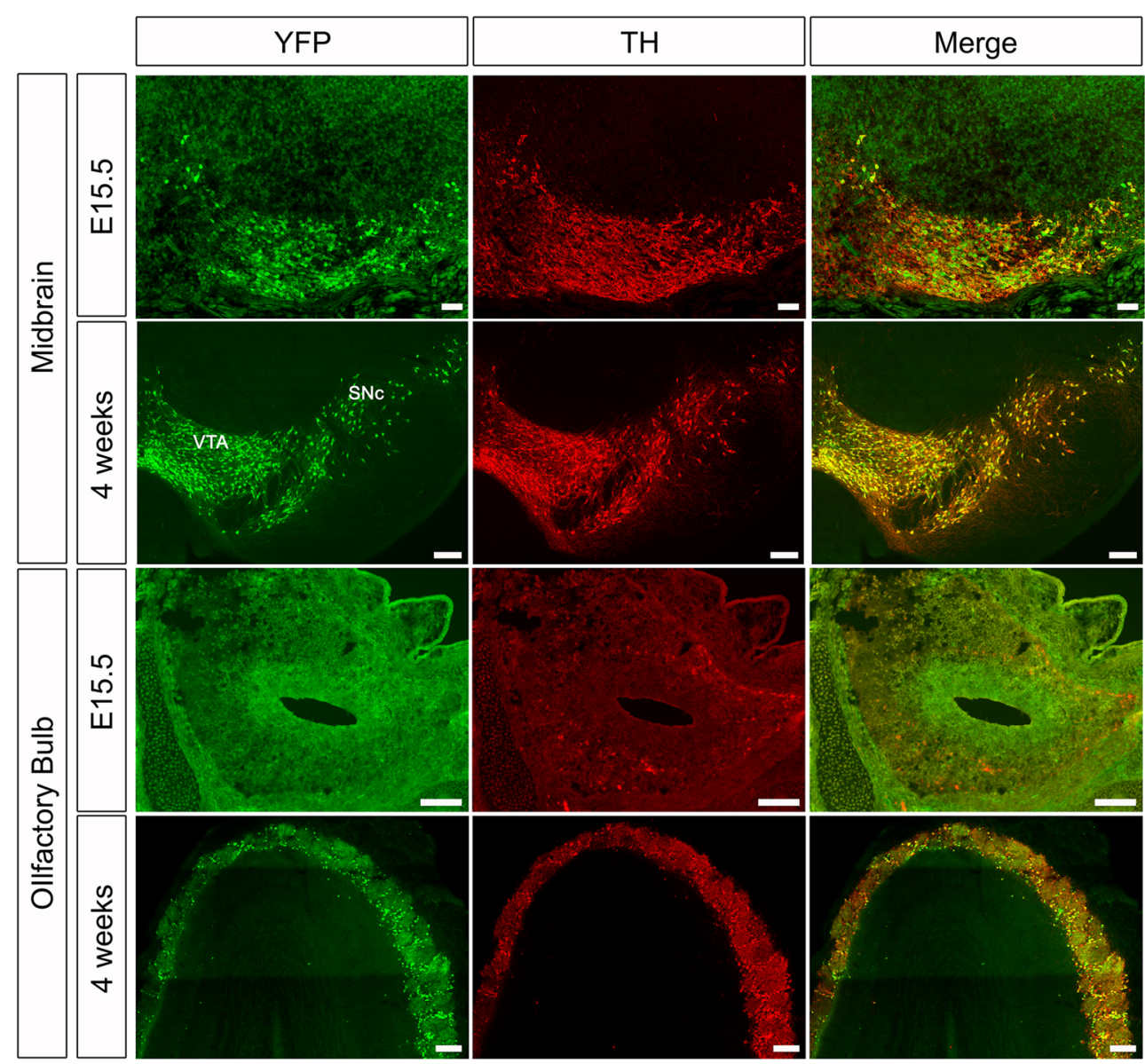

\section{Respiratory Chain Defects in OB DaNs of MitoPark Mice}

Loss of TFAM is accompanied by loss of mtDNA-encoded transcripts [32]. Consequently, respiratory chain defects become apparent in midbrain DaNs of 12-week-old MitoPark mice even before the onset of neurodegeneration [39]. In order to verify mitochondrial impairment in OB DaNs, a histochemical staining procedure for $\mathrm{COX} / \mathrm{SDH}$ activity was performed (Fig. 2e). Sporadically, COX-deficient cells were found in the GL of the OB in MitoPark mice; however, no COX-deficient cells were observed in any of the control animals. These data reveal that COX-deficiency also occurs in OB DaNs of MitoPark mice after inactivating the Tfam gene driven by Dat-Cre recombination.

\section{MitoPark Mice Exhibit Impaired Odor Detection}

To assess $\mathrm{OB}$ functionality in MitoPark mice, the olfactory behavior of control and MitoPark mice was investigated at different stages. In the buried pellet test, the latency to locate a food pellet, either hidden underneath the bedding or visible on the surface, respectively, was tested (for details of this widely used test, see supplementary methods). There was no significant difference in the latency time to detect the buried food pellet between MitoPark and control mice in any of the investigated ages (Fig. S1a). However, at the age of 30 weeks, MitoPark mice took significantly longer to find the visible pellet (Fig. S1b, control $15.14 \pm 5.21 \mathrm{~s}$, MitoPark $134.65 \pm 48.14 \mathrm{~s}$, two-way ANOVA, $P<0.05$ ), indicating that the severe motor impairment of 30 -weekold MitoPark mice may influence the result rather than showing exclusively an affected ability for odor detection.

In order to analyze odor discrimination as well as odor detection more independent from motor performance, the odor discrimination test was carried out in 20- and 30week-old MitoPark and control mice. Both non-social and social odors were presented consecutively three times each. Control animals revealed typical olfactory memory 


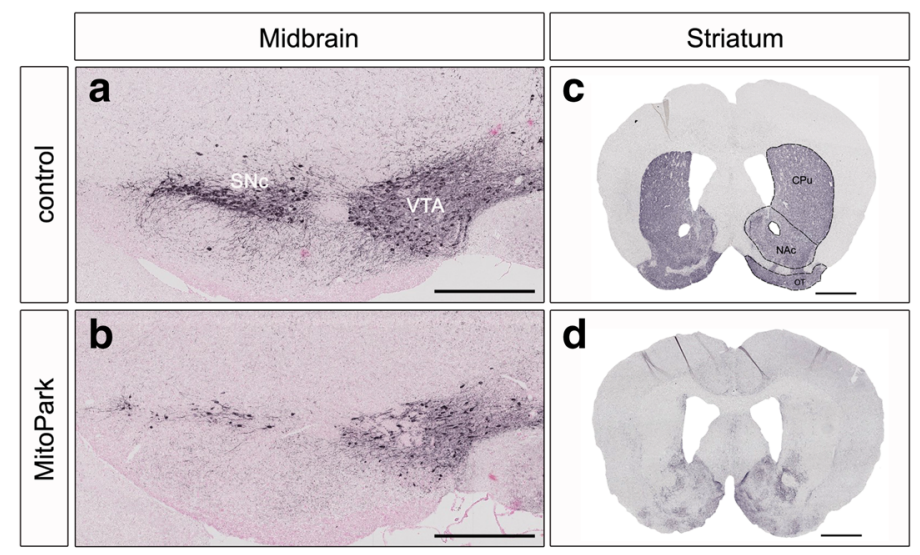

e

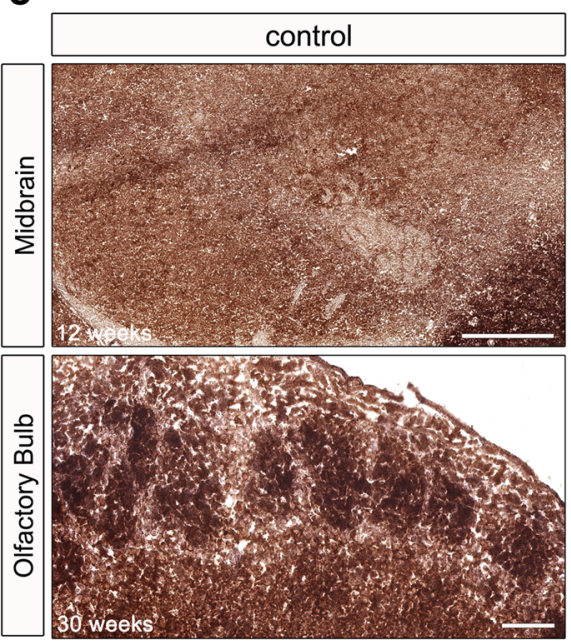

Fig. 2 Degeneration of midbrain DaNs and striatal projections in 30week-old MitoPark mice. a, b Tyrosine hydroxylase (TH) immunohistochemistry in the midbrain of MitoPark and age-matched control mice showing severe neuronal loss in the ventral tegmental area (VTA) and the substantia nigra pars compacta $(\mathrm{SNc})$. c, d Striatal staining presenting the reduction in TH-positive projection area in the nucleus accumbens (NAc), caudate putamen $(\mathrm{CPu})$, and the olfactory tubercle (OT). Three control and four MitoPark mice were analyzed. e COX-deficient DaNs in the $\mathrm{OB}$ of 30-week-old MitoPark mice. Neurons with reduced activity of cytochrome c oxidase (COX; brown) were unmasked by COX-SDH double staining (blue). In the midbrain, first COX-deficient cells become apparent at 12 weeks of age. Conversely, COX-deficient cells were found in the OB only after 30 weeks. f COX-deficient cells in the OB of 30week-old MitoPark mice. Quantitative analysis revealed a significantly higher number of blue cells in the OB of MitoPark mice when compared with control animals. Control mice: black bar $n=5$; MitoPark mice: red bar $n=4$. Scale bars: $500 \mu \mathrm{m}(\mathbf{a}, \mathbf{b}), 1 \mu \mathrm{mm}(\mathbf{c}, \mathbf{d})$, midbrain $500 \mu \mathrm{m}$, enlarged $50 \mu \mathrm{m}$; olfactory bulb $100 \mu \mathrm{m}$, enlarged $50 \mu \mathrm{m}$ (e) and discrimination. They presented habituation behavior to identical odors characterized by decreasing sniffing times as well as dishabituation to new odors with increasing sniffing times (Fig. 3a-d). For MitoPark mice, the time spent at the odors was dramatically reduced and they were not able to detect any of the presented odors. The time spent sniffing at the odor was significantly decreased in 20-week-old MitoPark mice for all odor types (Fig. 3c, d, water-1: two-way ANOVA, $P<0.001$, almond-1: $P<0.01$, banana-1: $P<0.01$, social 1-1: $P<0.001)$. Furthermore, 30 -week-old MitoPark mice spent no time at any of the presented odors (Fig. 3c, d). These results indicate that odor detection is fundamentally impaired in MitoPark mice.

\section{Reduction of Dopaminergic SCs in the OB of MitoPark Mice}

Soma size-based quantification was performed to examine the number of DaNs in the two main subpopulations (Fig. 4a, b). The number of SCs was higher compared with LACs in this area, in line with previously reported observations in wt mice [40]. The number of LACs and SCs did not differ between both groups at the age of 20 weeks, however, comparison of control and MitoPark mice showed a decreased number of SCs in 30week-old MitoPark (Fig. 4c, control: $351.25 \pm 26.06$, MitoPark: $228.67 \pm 22.50$, unpaired $t$ test, $P<0.01$ ). These results suggest that TFAM depletion in OB DaNs preferentially affects SC survival and that this may lead to the observed odor 
Fig. 3 Impaired odor detection in MitoPark mice. a-d Time spent sniffing at the odor by $20(\mathbf{a}, \mathbf{b})$ and 30-week-old (c, d) MitoPark (red squares) and control mice (black squares). 20 weeks: $(n=9$ 10 mice); 30 weeks: $(n=5-6$ mice)
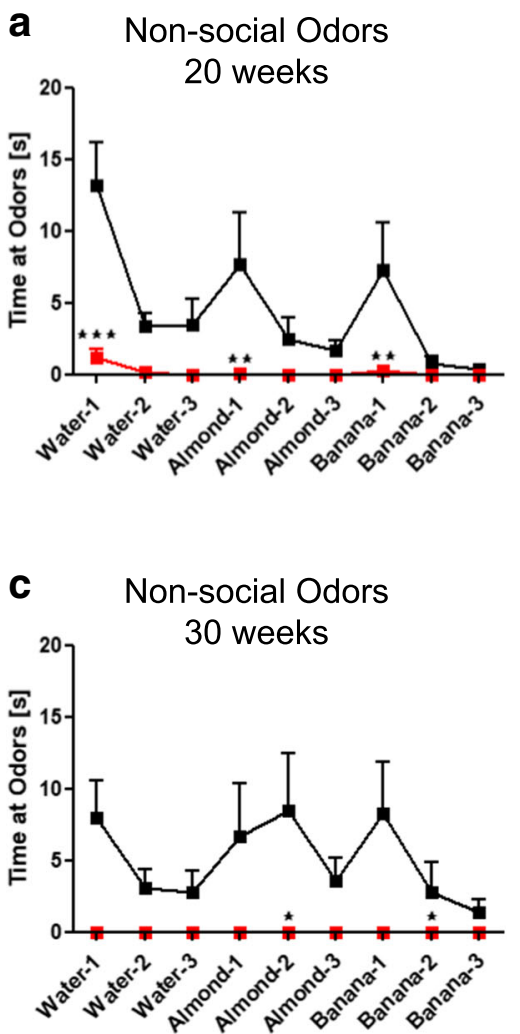
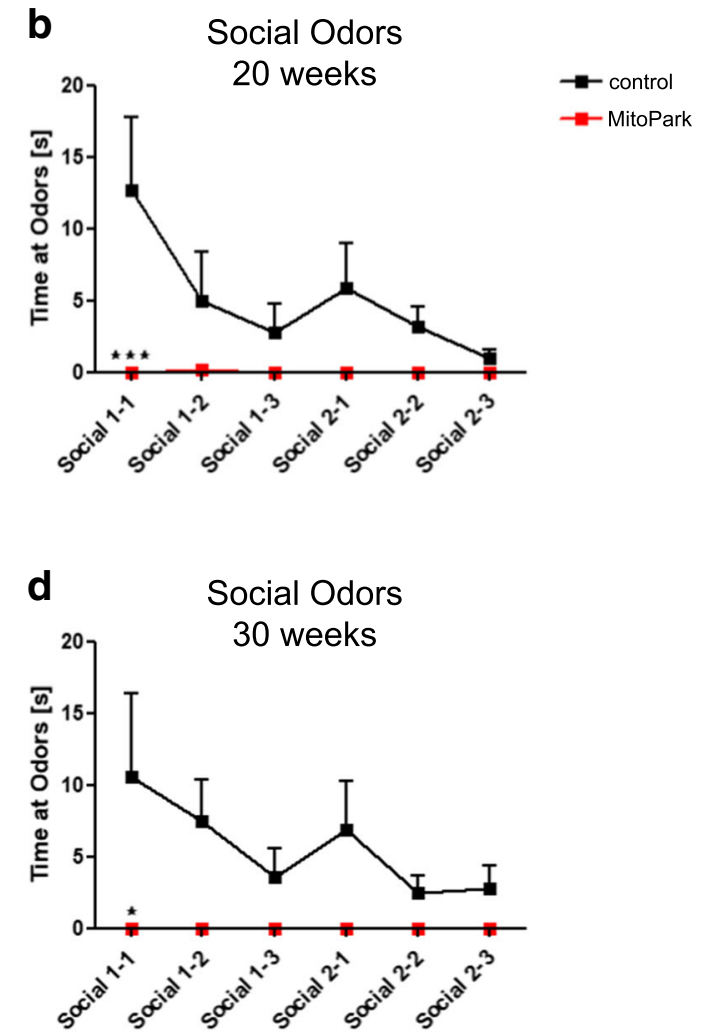

detection impairment. Alternatively, the continuous generation of progenitor cells could be affected and be the reason for the decreased SC number in MitoPark mice.

\section{Number of PAX6 ${ }^{+}$DaNs Is Not Altered in the OB of MitoPark Mice}

In contrast to LACs, SCs can be newly generated even postnatally. To investigate if the reduced number of SCs in
MitoPark mice is caused by an alteration in progenitor cell differentiation, PAX6 immunohistochemistry was performed in the OB (Fig. 5a). The transcription factor PAX6 is postnatally expressed in progenitor cells of the subventricular zone to mediate their dopaminergic fate [21-23, 41-43] and remains expressed in DaN progenitors after arriving in the OB [44-46]. In addition, PAX6 has recently been used by Höglinger and colleagues as a marker for neurogenic progenitors within the rostral migratory stream, which gives rise to

a

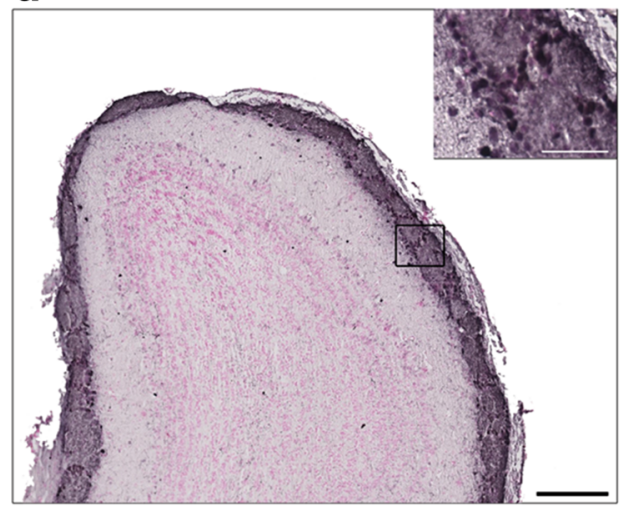

b

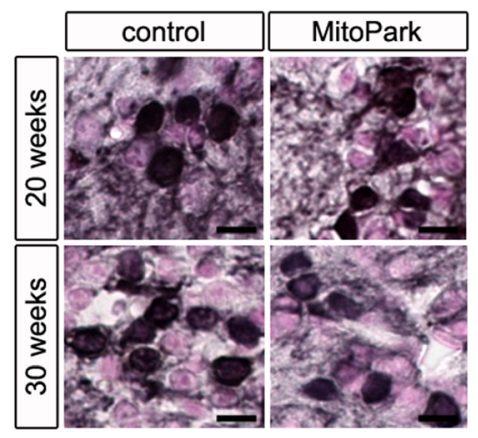

C

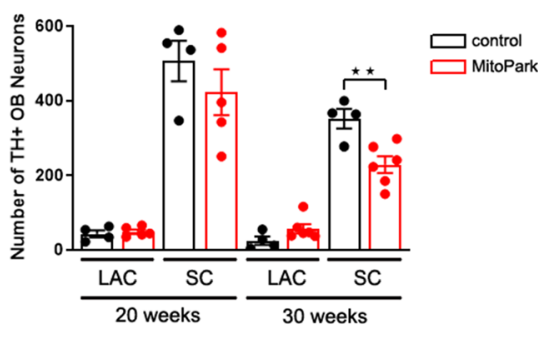

Fig. 4 Reduced number of SCs in the OB of MitoPark mice. a TH immunohistochemistry of the $\mathrm{OB}$ and the enlarged glomerular layer. $\mathbf{b}$ TH staining in MitoPark and age-matched control mice. $\mathbf{c}$ Quantification of DaN subpopulations showed a reduction in SCs in the OB of 30-weekold MitoPark mice. In addition, the amount of SCs was higher compared with LACs (20 weeks, control 506.25 \pm 54.54 SCs vs. $43.25 \pm 9.32$
LACs, one-way ANOVA, $P<0.0001$; MitoPark $422.20 \pm 61.70$ SCs vs. $49.00 \pm 5.81$ LACs, $P<0.0001 ; 30$ weeks, control $351.25 \pm 26.06$ SCs vs. $24.75 \pm 11.25$ LACs, $P<0.0001$; MitoPark $228.67 \pm 22.50$ SCs vs. $57.17 \pm 12.24$ LACs, $P>0.01)$. Control mice: black bars, 20 weeks $n=4,30$ weeks $n=4$; MitoPark mice: red bars, 20 weeks $n=5,30$ weeks $n=6$. Scale bars: $200 \mu \mathrm{m}$ and $50 \mu \mathrm{m}(\mathbf{a}), 10 \mu \mathrm{m}(\mathbf{b})$ 
a

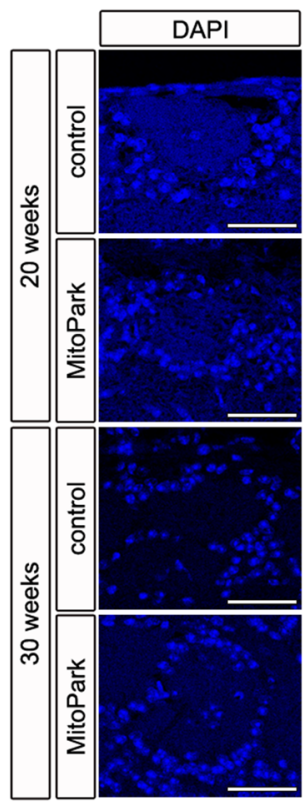

b

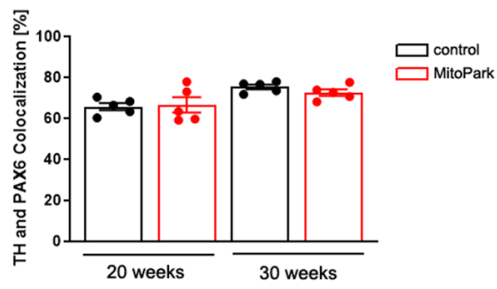

Fig. 5 No change in the amount of PAX6-expressing DaNs in the OB but in the amount of progenitor cells in the SVZ of MitoPark mice. a Merged TH and PAX6 immunofluorescent staining presents newborn OB DaNs in 20- and 30-week-old MitoPark and age-matched control mice. b Quantitative analysis of PAX6-expressing DaN number revealed no difference between MitoPark and control animals. Control mice: black bars, 20 weeks $n=5,30$ weeks $n=5$; MitoPark mice: red bars, 20 weeks $n=5$, 30 weeks $n=5$. Increase of PAX6-expressing progenitor cells in the SVZ

DaNs in the OB [47]. In contrast, PAX6 expression in cells already established during development decreased over time with no colocalization in neurons [48]. This makes PAX6 an ideal marker for adult-born DaNs in the mouse OB. The amount of TH-positive neurons also expressing PAX6 did not differ between MitoPark and control mice (Fig. 5b, 20 weeks: control $65.66 \pm 1.79 \%$, MitoPark $66.55 \pm 3.76 \%$; 30 weeks; control $75.30 \pm 1.17 \%$, MitoPark $72.54 \pm 1.59 \%$, one-way ANOVA, n.s.). These data reveal that DaN progenitor cell differentiation in the $\mathrm{OB}$ is not impaired in MitoPark mice.

\section{MitoPark Mice Show Increased Progenitor Cell Mobilization in the SVZ}

To further assess whether OB progenitor cell proliferation is affected earlier in the lineage of these neurons, PAX6 expression c

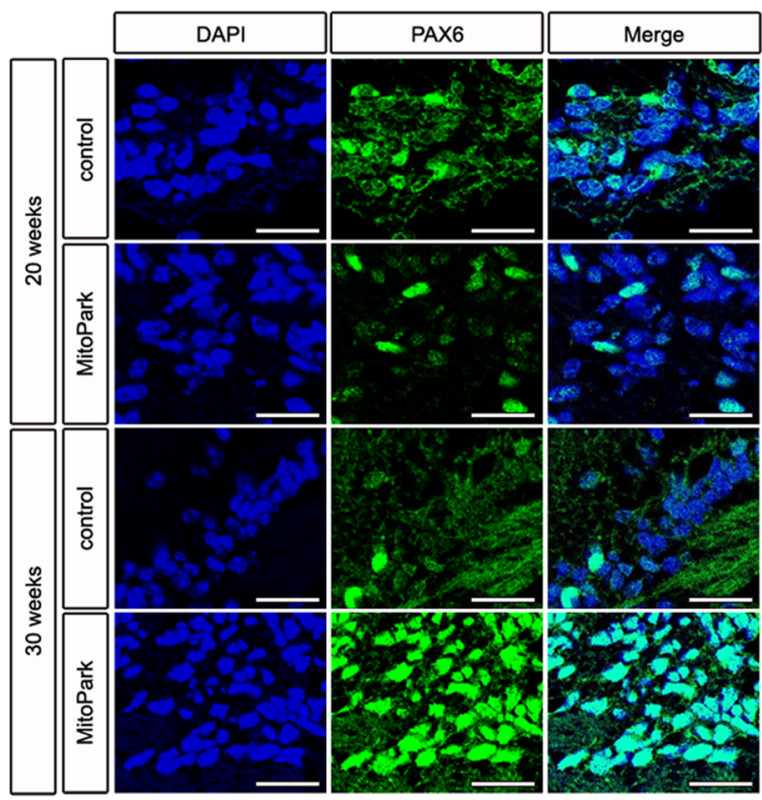

d

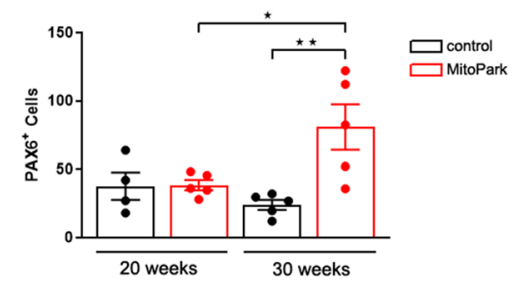

of MitoPark mice. c Combined nuclear (DAPI) and immunofluorescent PAX6 staining depicts the distribution of progenitor cells in MitoPark and age-matched control mice in the SVZ. d Quantitative analysis showed an enhanced number of progenitor cells in 30-week-old MitoPark mice. Control mice: black bars, 20 weeks $n=4,30$ weeks $n=5$; MitoPark mice: red bars, 20 weeks $n=5,30$ weeks $n=5$. Scale bars: $50 \mu \mathrm{m}(\mathbf{a}), 25 \mu \mathrm{m}$ (b)

in the dorsal SVZ was studied (Fig. 5c). At 20 weeks of age, there was no difference between MitoPark and control animals (Fig. 5d, control 37.69 $\pm 10.00 \%$, MitoPark 38.48 $\pm 3.71 \%$ ). However, 30-week-old MitoPark mice revealed a significantly increased number of PAX6-expressing cells when compared with 20-week-old MitoPark mice and age-matched control animals (MitoPark: 20 weeks $38.48 \pm 3.71 \%$, 30 weeks $80.80 \pm$ $16.63 \%$, one-way ANOVA, $P<0.05 ; 30$ weeks: control $24.02 \pm 3.65 \%$, one-way ANOVA, $P<0.01)$. These results indicate an enhanced mobilization of progenitor cells in the dorsal SVZ, probably induced by the decline of SCs in the OB.

\section{Discussion}

In contrast to the midbrain, OB DaNs reveal no Dat-Cre expression during embryonic development (Fig. 1). Moreover, 
RNA in situ hybridization data [38] suggest that the Dat gene only starts to be expressed in OB DaNs in the second postnatal week. Subsequently, cells with respiratory chain deficits become apparent in OB DaNs only in 30-week-old MitoPark mice (Fig. 2e), i.e. 28 weeks after TFAM inactivation at around P14, whereas midbrain DaNs uniformly present COX-deficiency already after 12 weeks of age [39], i.e. 13 weeks after TFAM inactivation at around E15.5. This reveals a surprisingly different response of different $\mathrm{DaN}$ populations to inactivation of mitochondrial gene expression.

To inspect MitoPark mice for OB functionality, olfactory behavior was investigated. The widely used buried pellet test did not reveal olfactory dysfunction in MitoPark mice (Fig. S1A-B), but this may rather be a consequence of the motor impairment at 30 weeks of age. It further has to be noted that the outcome of the buried food pellet test does not exclusively depend on the detection of odors. As a consequence of the bradykinesia, MitoPark mice have to be supplied with moistened food pellets on the surface of the bedding from an age of 15 weeks onward. MitoPark mice are thereby trained to seek for food at the surface by housing conditions. Furthermore, the animals were not food deprived since this would be ethically not permitted with MitoPark mice. In fact, these animals could even suffer from an enhanced desire for food since they start losing weight at 20 weeks of age [49]. Thereby, the buried food pellet test might not be an appropriate test to analyze odor detection impairment in MitoPark mice.

It has further been shown that OB DaNs are involved in olfactory discrimination $[7,50,51]$. In order to analyze discrimination as wells as detection more independently from motor performance and food seeking, the odor discrimination test was performed (Fig. 3a-d). Already 20-week-old MitoPark mice show sharply reduced times spent at all presented odors. After 30 weeks of age, no sniffing time is observed at all, indicating a severe impairment in fundamental odor detection. Noteworthy, the ability of MitoPark mice to move was still sufficient to localize the presented odors (see video sequences in Videos 1 and 2).

In order to investigate the number of DaNs in the GL, soma size-based quantification was performed. Intriguingly, only a reduced number of SCs is found in 30-week-old MitoPark mice, whereas LACs were unaffected (Fig. 4c). In the midbrain of MitoPark mice, both SNc and VTA DaNs present mitochondrial dysfunction at 12 weeks of age, followed by progressive neurodegeneration, with $\mathrm{SNc}$ neurons being more affected [32]. This selective vulnerability is one hallmark for $\mathrm{PD}$ in patients [52] and raises the question which cell typespecific factors render $\mathrm{SNc} \mathrm{DaNs}$ vulnerable to mitochondrial dysfunction. Much research has been conducted characterizing neuroanatomical as well as electrophysiological properties of midbrain and OB DaNs. On the one hand, complex axonal morphology might play an essential role regarding the time course of degeneration. SNc DaNs show an extremely large arborization with an estimated number of 100.000-250.000 synapses per neuron [53] compared with both VTA and OB DaNs, with the SCs even being anaxonic [14]. The extended branching results in an extreme bioenergetic demand, leaving SNc DaNs working on a tight energy budget [54], especially when facing additional stressors, such as mitochondrial dysfunction. On the other hand, all three DaN populations are characterized as autonomous pacemakers $[40,55,56]$. The pacemaking machinery of VTA and OB DaNs is mainly driven by a persistent sodium current $[40,57,58]$. Conversely, $\mathrm{SNc}$ pacemaker activity is associated with $\mathrm{Ca}^{2+}$ influx through plasma membrane $\mathrm{Ca}_{\mathrm{v}} 1.3$ channels, postulated to cause oxidant stress in the mitochondrial compartment $[59,60]$. Combined with a low intrinsic calcium buffering capacity, mitochondrial dysfunction leads to an oxidized RedOxsystem and hyperpolarized membrane potential in mitochondria of SNc DaNs, which thereby causes neuron death [39].

SCs and LACs differ in various aspects, including morphology, functionality, and neurogenic potential. SCs are anaxonic and thereby generate somatic action potentials with a low firing rate. In contrast, the wide-branching LACs do have an axon and a high firing frequency [19]. SCs are typically type 1 periglomerular cells [61-65]. They receive olfactory nerve and dendrodendritic synapses, which, in turn, lead to the inhibition of mitral cells [66], the principal output neurons of the OB. A reduced number or functional changes of SCs in MitoPark mice could thereby cause temporal shifts in mitral cell activity, previously shown to impair olfactoryrelated behaviors [67].

In addition, anaxonic SCs are even continuously formed via adult neurogenesis, whereas LACs are exclusively established during embryonic development [15-19]. Consequently, affected neuronal replenishment of SCs could be the explanation for the reduced number of SCs. OB progenitor cells are created in the SVZ of the lateral ventricles and tangentially migrate along the rostral migratory stream before they enter the OB [68]. Those progenitor cells are characterized by the expression of PAX6. Interestingly, MitoPark mice reveal an increased mobilization of PAX6-expressing cells in the SVZ at 30 weeks of age (Fig. $5 \mathrm{c}, \mathrm{d}$ ), indicating a potential compensatory upregulation of progenitor cells. However, the amount of PAX6expressing DaNs in the OB is stable (Fig. 5a, b). This gives reason to suppose that either the death rate of SCs is so high that the number of replenished neurons in the OB cannot compensate for this or that the upregulation of progenitor cells in the SVZ is not a direct cause of the reduced SC number. In addition, even though the time line of RMS migration is very well established, there is no evidence to when DAT expression is initiated in OB progenitor cells. Potentially, new but not fully matured neurons are more vulnerable to the TFAM knockout due to early DAT expression and die even before reaching the $\mathrm{OB}$. 
Besides the reduced number of SCs, it is likely that the severe olfactory dysfunction is caused by other factors. So far no direct link between the midbrain and the $\mathrm{OB}$ could be demonstrated $[69,70]$. However, a recent study discovered the existence of axonal projections from SNc DaNs to the $\mathrm{OB}$ and the ablation of this connectivity resulted in impaired olfactory perception [71]. At 30 weeks of age, MitoPark mice reveal a severe degeneration of SNc DaNs (Fig. 2b). Therefore, the absence of these nigro-bulbar connections could contribute to the olfactory dysfunction in MitoPark mice. More precisely, the loss of SNc-OB projections might lead to $\mathrm{Ca}^{2+}$-induced hyperactivity of mitral cells caused by the missing dopaminergic inhibition, as recently demonstrated in a 6-OHDA induced PD mouse model [72]. However, Zhang et al. did not show any data concerning the dopaminergic projection area in the striatum after partial depletion of SNc DaNs, though striatal denervation is likewise affecting olfactory behavior [73]. Since MitoPark mice reveal the degeneration of SNc DaNs as well as corresponding striatal fibers, we postulate that, beside the decreased number of SCs, olfactory dysfunction might be caused by the degeneration of the complete nigro-striatal system. Moreover, the loss of the nigrostriatal system might be the reason for the increased mobilization of progenitor cells in the SVZ observed in 30week-old MitoPark mice, as shown likewise after 6OHDA lesioning [74]. Noteworthy, DaNs from the VTA innervate the SVZ and are involved in proliferation of progenitor cells [75]. Increased progenitor cell mobilization may thereby also have its reason in a compensatory upregulation due to the loss of VTA DaNs. More importantly, the SNc and the VTA as well as the striatum further possess dopaminergic projections to higher olfactory brain regions, such as the piriform cortex and the olfactory tubercle [76-78]. Therefore, a prospective disconnection between the nigro-striatal system as well as the VTA and higher olfactory centers might importantly contribute to the impaired olfactory-related behavior in MitoPark mice.

Apart from the dopaminergic system, serotonin could also play a role in the olfactory dysfunction of MitoPark mice. Serotonergic neurons from the raphe nuclei are innervating all layers of the OB [79-81] and deafferentiation of corresponding fibers causes anosmia and OB atrophy [82]. Furthermore, mice that are deficient for PTEN-induced kinase 1 (PINK1), mutations of which make up 1-2\% of the familiar forms of $\mathrm{PD}$, possess a decreased serotonergic innervation in the GL, leading to an impaired olfactory behavior [83]. Besides the SNc, the raphe nuclei are also affected in PD patients, with a loss of serotonergic neurons and its projections $[84,85]$. However, altered serotonin transporter activities in PD patients failed to correlate with olfactory dysfunction [86], leaving the role of serotonergic neurons in PD-related anosmia unclear.
Our data provide new insights into olfactory dysfunction and adaptations of adult neurogenesis in response to genetic depletion of the dopaminergic system. Furthermore, we show that dopaminergic neurons located in the olfactory bulb reveal a high robustness towards mitochondrial impairment, in striking contrast to their midbrain counterparts.

Acknowledgments We acknowledge Robin Wolter for his help with cutting the paraffin-embedded sections of the OB. We would like to thank N.G. Larsson (Karolinska Institute, Stockholm) for generously providing mouse strains for the generation of MitoPark mice.

Funding Information Open Access funding provided by Projekt DEAL. K.M.R. was supported by the graduate program in Pharmacology and Experimental Therapeutics at the University of Cologne, which is financially and scientifically supported by Bayer and by Köln Fortune; K.M.R., T.P., and R.W. were supported by the Deutsche Forschungsgemeinschaft (DFG, SFB 1218/TP B07). R.J.W. was also funded by the DFG (Cologne Excellence Cluster on Cellular Stress Responses in Aging-associated Diseases - CECAD). S.B. was supported by the DFG (BL 767/3-1; Heisenberg Program BL 767/2-1, BL767/4-1, BL 767/5-1; SFB1089/TP B05) and the Maria von Linden-Program (University of Bonn). Work from M.R. was supported by the DFG (RO4046/2-1 and /2-2, Emmy Noether Program; GRK2416).

Open Access This article is licensed under a Creative Commons Attribution 4.0 International License, which permits use, sharing, adaptation, distribution and reproduction in any medium or format, as long as you give appropriate credit to the original author(s) and the source, provide a link to the Creative Commons licence, and indicate if changes were made. The images or other third party material in this article are included in the article's Creative Commons licence, unless indicated otherwise in a credit line to the material. If material is not included in the article's Creative Commons licence and your intended use is not permitted by statutory regulation or exceeds the permitted use, you will need to obtain permission directly from the copyright holder. To view a copy of this licence, visit http://creativecommons.org/licenses/by/4.0/.

\section{References}

1. Tysnes O-B, Storstein A (2017) Epidemiology of Parkinson's disease. J Neural Transm 124:901-905. https://doi.org/10.1007/ s00702-017-1686-y

2. Michel PP, Hirsch EC, Hunot S (2016) Understanding dopaminergic cell death pathways in Parkinson disease. Neuron. 90:675-691. https://doi.org/10.1016/j.neuron.2016.03.038

3. Haehner A, Hummel T, Reichmann H (2009) Olfactory dysfunction as a diagnostic marker for Parkinson's disease. Expert Rev Neurother 9:1773-1779. https://doi.org/10.1586/ern.09.115

4. Doty RL (2012) Olfaction in Parkinson's disease and related disorders. Neurobiol Dis 46:527-552. https://doi.org/10.1016/j.nbd. 2011.10.026

5. Klingelhoefer L, Reichmann H (2015) Pathogenesis of Parkinson disease-the gut-brain axis and environmental factors. Nat Rev Neurol 11:625-636. https://doi.org/10.1038/nrneurol.2015.197

6. Cave JW, Baker H (2009) Dopamine systems in the forebrain. Adv Exp Med Biol:15-35

7. Tillerson JL, Caudle WM, Parent JM, Gong C, Schallert T, Miller GW (2006) Olfactory discrimination deficits in mice lacking the dopamine transporter or the D2 dopamine receptor. Behav Brain Res 172:97-105. https://doi.org/10.1016/j.bbr.2006.04.025 
8. Escanilla O, Yuhas C, Marzan D, Linster C (2009) Dopaminergic modulation of olfactory bulb processing affects odor discrimination learning in rats. Behav Neurosci 123:828-833. https://doi.org/10. 1037/a0015855

9. Berkowicz DA, Trombley PQ (2000) Dopaminergic modulation at the olfactory nerve synapse. Brain Res 855:90-99

10. Wachowiak M, McGann JP, Heyward PM, Shao Z, Puche AC, Shipley MT (2005) Inhibition corrected of olfactory receptor neuron input to olfactory bulb glomeruli mediated by suppression of presynaptic calcium influx. J Neurophysiol 94:2700-2712. https:// doi.org/10.1152/jn.00286.2005

11. Nagayama S, Homma R, Imamura F (2014) Neuronal organization of olfactory bulb circuits. Front Neural Circuits 8:98. https://doi. org/10.3389/fncir.2014.00098

12. Banerjee A, Marbach F, Anselmi F, Koh MS, Davis MB, Garcia da Silva $P$ et al (2015) An interglomerular circuit gates glomerular output and implements gain control in the mouse olfactory bulb. Neuron. 87:193-207. https://doi.org/10.1016/j.neuron.2015.06.019

13. Liu S, Plachez C, Shao Z, Puche A, Shipley MT (2013) Olfactory bulb short axon cell release of GABA and dopamine produces a temporally biphasic inhibition-excitation response in external tufted cells. J Neurosci 33:2916-2926. https://doi.org/10.1523/ JNEUROSCI.3607-12.2013

14. Pignatelli A, Belluzzi O (2017) Dopaminergic neurones in the main olfactory bulb: an overview from an electrophysiological perspective. Front Neuroanat 11:7. https://doi.org/10.3389/fnana.2017. 00007

15. Kosaka K, Hama K, Nagatsu I, Wu J-Y, Ottersen OP, StormMathisen J, Kosaka T (1987) Postnatal development of neurons containing both catecholaminergic and GABAergic traits in the rat main olfactory bulb. Brain Res 403:355-360. https://doi.org/ 10.1016/0006-8993(87)90075-8

16. Vergano-Vera E, Yusta-Boyo MJ, de Castro F, Bernad A, de Pablo F, Vicario-Abejón C (2006) Generation of GABAergic and dopaminergic interneurons from endogenous embryonic olfactory bulb precursor cells. Development. 133:4367-4379

17. Bovetti S, Veyrac A, Peretto P, Fasolo A, de Marchis S (2009) Olfactory enrichment influences adult neurogenesis modulating GAD67 and plasticity-related molecules expression in newborn cells of the olfactory bulb. PLoS One 4:e6359. https://doi.org/10. 1371/journal.pone.0006359

18. Kosaka T, Kosaka K (2009) Two types of tyrosine hydroxylase positive GABAergic juxtaglomerular neurons in the mouse main olfactory bulb are different in their time of origin. Neurosci Res 64: 436-441. https://doi.org/10.1016/j.neures.2009.04.018

19. Galliano E, Franzoni E, Breton M, Chand AN, Byrne DJ, Murthy VN, Grubb MS (2018) Embryonic and postnatal neurogenesis produce functionally distinct subclasses of dopaminergic neuron. Elife 7. https://doi.org/10.7554/eLife.32373

20. Fiorelli R, Azim K, Fischer B, Raineteau O (2015) Adding a spatial dimension to postnatal ventricular-subventricular zone neurogenesis. Development. 142:2109-2120. https://doi.org/10. 1242/dev.119966

21. Merkle FT, Mirzadeh Z, Alvarez-Buylla A (2007) Mosaic organization of neural stem cells in the adult brain. Science. 317:381-384. https://doi.org/10.1126/science.1144914

22. Young KM, Fogarty M, Kessaris N, Richardson WD (2007) Subventricular zone stem cells are heterogeneous with respect to their embryonic origins and neurogenic fates in the adult olfactory bulb. J Neurosci 27:8286-8296. https://doi.org/10.1523/ JNEUROSCI.0476-07.2007

23. Brill MS, Snapyan M, Wohlfrom H, Ninkovic J, Jawerka M, Mastick GS, Ashery-Padan R, Saghatelyan A et al (2008) A dlx2and pax6-dependent transcriptional code for periglomerular neuron specification in the adult olfactory bulb. J Neurosci 28:6439-6452. https://doi.org/10.1523/JNEUROSCI.0700-08.2008
24. Codega P, Silva-Vargas V, Paul A, Maldonado-Soto AR, Deleo AM, Pastrana E, Doetsch F (2014) Prospective identification and purification of quiescent adult neural stem cells from their in vivo niche. Neuron. 82:545-559. https://doi.org/10.1016/j.neuron.2014. 02.039

25. Bonaguidi MA, Stadel RP, Berg DA, Sun J, Ming G-1, Song H (2016) Diversity of neural precursors in the adult mammalian brain. Cold Spring Harb Perspect Biol 8:a018838. https://doi.org/10. 1101/cshperspect.a018838

26. Mundiñano I-C, Caballero M-C, Ordóñez C, Hernandez M, DiCaudo C, Marcilla I, Erro ME, Tuñon MT et al (2011) Increased dopaminergic cells and protein aggregates in the olfactory bulb of patients with neurodegenerative disorders. Acta Neuropathol 122:61-74. https://doi.org/10.1007/s00401-0110830-2

27. Huisman E, Uylings HBM, Hoogland PV (2008) Gender-related changes in increase of dopaminergic neurons in the olfactory bulb of Parkinson's disease patients. Mov Disord 23:1407-1413. https:// doi.org/10.1002/mds.22009

28. Cave JW, Fujiwara N, Weibman AR, Baker H (2016) Cytoarchitectural changes in the olfactory bulb of Parkinson's disease patients. NPJ Parkinsons Dis 2:16011. https://doi.org/10. 1038/npjparkd.2016.11

29. Dölle C, Flønes I, Nido GS, Miletic H, Osuagwu N, Kristoffersen S, Lilleng PK, Larsen JP et al (2016) Defective mitochondrial DNA homeostasis in the substantia nigra in Parkinson disease. Nat Commun 7:13548. https://doi.org/10.1038/ncomms13548

30. Campbell CT, Kolesar JE, Kaufman BA (1819) Mitochondrial transcription factor A regulates mitochondrial transcription initiation, DNA packaging, and genome copy number. Biochim Biophys Acta 2012:921-929. https://doi.org/10.1016/j.bbagrm.2012.03.002

31. Gustafsson CM, Falkenberg M, Larsson N-G (2016) Maintenance and expression of mammalian mitochondrial DNA. Annu Rev Biochem 85:133-160. https://doi.org/10.1146/annurev-biochem060815-014402

32. Ekstrand MI, Terzioglu M, Galter D, Zhu S, Hofstetter C, Lindqvist E, Thams S, Bergstrand A et al (2007) Progressive parkinsonism in mice with respiratory-chain-deficient dopamine neurons. PNAS. 104:1325-1330. https://doi.org/10.1073/pnas.0605208103

33. Ekstrand MI, Galter D (2009) The MitoPark mouse - an animal model of Parkinson's disease with impaired respiratory chain function in dopamine neurons. Parkinsonism Relat Disord 15:S185S188. https://doi.org/10.1016/S1353-8020(09)70811-9

34. Sciacco M, Bonilla E (1996) Cytochemistry and immunocytochemistry of mitochondria in tissue sections. Methods Enzymol 264: 509-521

35. Bonzano S, Bovetti S, Gendusa C, Peretto P, de Marchis S (2016) Adult born olfactory bulb dopaminergic interneurons: molecular determinants and experience-dependent plasticity. Front Neurosci 10:189. https://doi.org/10.3389/fnins.2016.00189

36. Srinivas S, Watanabe T, Lin C-S, William CM, Tanabe Y, Jessell TM, Costantini F (2001) Cre reporter strains produced by targeted insertion of EYFP and ECFP into the ROSA26 locus. BMC Dev Biol 1:4. https://doi.org/10.1186/1471-213X-1-4

37. Vaswani AR, Weykopf B, Hagemann C, Fried H-U, Brüstle O, Blaess S (2019) Correct setup of the substantia nigra requires Reelin-mediated fast, laterally-directed migration of dopaminergic neurons. Elife 8. https://doi.org/10.7554/eLife.41623

38. Allen Institute. Allen developing brain atlas. 2015. http:// developingmouse.brain-map.org. Accessed 10th July 2019.

39. Ricke KM, Paß T, Kimoloi S, Fährmann K, Jüngst C, Schauss A, Baris OR, Aradjanski M et al (2020) Mitochondrial dysfunction combined with high calcium load leads to impaired antioxidant defense underlying the selective loss of nigral dopaminergic neurons. J Neurosci 40:1975-1986. https://doi.org/10.1523/ JNEUROSCI.1345-19.2019 
40. Pignatelli A, Kobayashi K, Okano H, Belluzzi O (2005) Functional properties of dopaminergic neurones in the mouse olfactory bulb. J Physiol 564:501-514. https://doi.org/10.1113/jphysiol.2005. 084632

41. Hack MA, Saghatelyan A, de Chevigny A, Pfeifer A, AsheryPadan R, Lledo P-M, Götz M (2005) Neuronal fate determinants of adult olfactory bulb neurogenesis. Nat Neurosci 8:865-872. https://doi.org/10.1038/nn1479

42. de Chevigny A, Core N, Follert P, Wild S, Bosio A, Yoshikawa K, Cremer H, Beclin C (2012) Dynamic expression of the prodopaminergic transcription factors Pax6 and Dlx2 during postnatal olfactory bulb neurogenesis. Front Cell Neurosci 6:6. https://doi. org/10.3389/fncel.2012.00006

43. Curto GG, Nieto-Estévez V, Hurtado-Chong A, Valero J, Gómez C, Alonso JR, Weruaga E, Vicario-Abejón C (2014) Pax6 is essential for the maintenance and multi-lineage differentiation of neural stem cells, and for neuronal incorporation into the adult olfactory bulb. Stem Cells Dev 23:2813-2830. https://doi.org/10.1089/scd. 2014.0058

44. Kohwi M, Osumi N, Rubenstein JLR, Alvarez-Buylla A (2005) Pax6 is required for making specific subpopulations of granule and periglomerular neurons in the olfactory bulb. J Neurosci 25: 6997-7003. https://doi.org/10.1523/JNEUROSCI.1435-05.2005

45. Ninkovic J, Pinto L, Petricca S, Lepier A, Sun J, Rieger MA, Schroeder T, Cvekl A et al (2010) The transcription factor Pax6 regulates survival of dopaminergic olfactory bulb neurons via crystallin $\alpha$ A. Neuron. 68:682-694. https://doi.org/10.1016/j. neuron.2010.09.030

46. Agoston Z, Heine P, Brill MS, Grebbin BM, Hau A-C, KallenbornGerhardt W, Schramm J, Gotz M et al (2014) Meis2 is a Pax6 cofactor in neurogenesis and dopaminergic periglomerular fate specification in the adult olfactory bulb. Development. 141:28-38. https://doi.org/10.1242/dev.097295

47. Schweyer K, Rüschoff-Steiner C, Arias-Carrión O, Oertel WH, Rösler TW, Höglinger GU (2019) Neuronal precursor cells with dopaminergic commitment in the rostral migratory stream of the mouse. Sci Rep 9:13359. https://doi.org/10.1038/s41598-01949920-5

48. Duan D, Fu Y, Paxinos G, Watson C (2013) Spatiotemporal expression patterns of Pax6 in the brain of embryonic, newborn, and adult mice. Brain Struct Funct 218:353-372. https://doi.org/10. 1007/s00429-012-0397-2

49. Galter D, Pernold K, Yoshitake T, Lindqvist E, Hoffer B, Kehr J, Larsson NG, Olson L (2010) MitoPark mice mirror the slow progression of key symptoms and L-DOPA response in Parkinson's disease. Genes Brain Behav 9:173-181. https://doi.org/10.1111/j. 1601-183X.2009.00542.x

50. Kruzich PJ, Grandy DK (2004) Dopamine D2 receptors mediate two-odor discrimination and reversal learning in C57BL/6 mice. BMC Neurosci 5:12. https://doi.org/10.1186/1471-2202-5-12

51. Pavlis M, Feretti C, Levy A, Gupta N, Linster C (2006) 1-DOPA improves odor discrimination learning in rats. Physiol Behav 87: 109-113. https://doi.org/10.1016/j.physbeh.2005.09.011

52. Giguère N, Burke Nanni S, Trudeau L-E (2018) On cell loss and selective vulnerability of neuronal populations in Parkinson's disease. Front Neurol 9:455. https://doi.org/10.3389/fneur.2018. 00455

53. Bolam JP, Pissadaki EK (2012) Living on the edge with too many mouths to feed: why dopamine neurons die. Mov Disord 27:14781483. https://doi.org/10.1002/mds.25135

54. Pacelli C, Giguère N, Bourque M-J, Lévesque M, Slack RS, Trudeau L-É (2015) Elevated mitochondrial bioenergetics and axonal arborization size are key contributors to the vulnerability of dopamine neurons. Curr Biol 25:2349-2360. https://doi.org/10. 1016/j.cub.2015.07.050
55. Grace AA (2010) Dopamine system dysregulation by the ventral subiculum as the common pathophysiological basis for schizophrenia psychosis, psychostimulant abuse, and stress. Neurotox Res 18: 367-376. https://doi.org/10.1007/s12640-010-9154-6

56. Dragicevic E, Schiemann J, Liss B (2015) Dopamine midbrain neurons in health and Parkinson's disease: emerging roles of voltage-gated calcium channels and ATP-sensitive potassium channels. Neurosci. 284:798-814. https://doi.org/10.1016/j. neuroscience.2014.10.037

57. Khaliq ZM, Bean BP (2010) Pacemaking in dopaminergic ventral tegmental area neurons: depolarizing drive from background and voltage-dependent sodium conductances. J Neurosci 30:74017413. https://doi.org/10.1523/JNEUROSCI.0143-10.2010

58. Pignatelli A, Iseppe AF, Gambardella C, Borin M, Belluzzi O (2012) editors. Pacemaker currents in dopaminergic neurones of the mice olfactory bulb: Intech Open

59. Guzman JN, Sanchez-Padilla J, Wokosin D, Kondapalli J, Ilijic E, Schumacker PT, Surmeier DJ (2010) Oxidant stress evoked by pacemaking in dopaminergic neurons is attenuated by DJ-1. Nature. 468:696-700. https://doi.org/10.1038/nature09536

60. Guzman JN, Ilijic E, Yang B, Sanchez-Padilla J, Wokosin D, Galtieri D, Kondapalli J, Schumacker PT et al (2018) Systemic isradipine treatment diminishes calcium-dependent mitochondrial oxidant stress. J Clin Invest 128:2266-2280. https://doi.org/10. 1172/JCI95898

61. Koster NL, Norman AB, Richtand NM, Nickell WT, Puche AC, Pixley SK, Shipley MT (1999) Olfactory receptor neurons express D2 dopamine receptors. J Comp Neurol 411:666-673

62. Wachowiak M, Cohen LB (1999) Presynaptic inhibition of primary olfactory afferents mediated by different mechanisms in lobster and turtle. J Neurosci 19:8808-8817

63. Ennis M, Zhou FM, Ciombor KJ, Aroniadou-Anderjaska V, Hayar A, Borrelli E, Zimmer LA, Margolis F et al (2001) Dopamine D2 receptor-mediated presynaptic inhibition of olfactory nerve terminals. J Neurophysiol 86:2986-2997. https://doi.org/10.1152/jn. 2001.86.6.2986

64. Parrish-Aungst S, Shipley MT, Erdelyi F, Szabo G, Puche AC (2007) Quantitative analysis of neuronal diversity in the mouse olfactory bulb. J Comp Neurol 501:825-836. https://doi.org/10. 1002/cne.21205

65. Maher BJ, Westbrook GL (2008) Co-transmission of dopamine and GABA in periglomerular cells. J Neurophysiol 99:1559-1564. https://doi.org/10.1152/jn.00636.2007

66. Wachowiak M, Shipley MT (2006) Coding and synaptic processing of sensory information in the glomerular layer of the olfactory bulb. Semin Cell Dev Biol 17:411-423. https://doi.org/10.1016/j. semcdb.2006.04.007

67. Rebello MR, McTavish TS, Willhite DC, Short SM, Shepherd GM, Verhagen JV (2014) Perception of odors linked to precise timing in the olfactory system. PLoS Biol 12:e1002021. https://doi.org/10. 1371/journal.pbio.1002021

68. Sun W, Kim H, Moon Y (2010) Control of neuronal migration through rostral migration stream in mice. Anat Cell Biol 43:269 279. https://doi.org/10.5115/acb.2010.43.4.269

69. McLean JH, Shipley MT (1988) Postmitotic, postmigrational expression of tyrosine hydroxylase in olfactory bulb dopaminergic neurons. J Neurosci 8:3658-3669

70. Shipley MT, Ennis M (1996) Functional organization of olfactory system. J Neurobiol 30:123-176. https://doi.org/10.1002/(SICI) 1097-4695(199605)30:1<123::AID-NEU11>3.0.CO;2-N

71. Höglinger GU, Alvarez-Fischer D, Arias-Carrión O, Djufri M, Windolph A, Keber U, Borta A, Ries V et al (2015) A new dopaminergic nigro-olfactory projection. Acta Neuropathol 130:333348. https://doi.org/10.1007/s00401-015-1451-y

72. Zhang W, Sun C, Shao Y, Zhou Z, Hou Y, Li A (2019) Partial depletion of dopaminergic neurons in the substantia nigra impairs 
olfaction and alters neural activity in the olfactory bulb. Sci Rep 9: 254. https://doi.org/10.1038/s41598-018-36538-2

73. Valle-Leija P, Drucker-Colín R (2014) Unilateral olfactory deficit in a hemiparkinson's disease mouse model. Neuroreport. 25:948953. https://doi.org/10.1097/WNR.0000000000000218

74. Winner B, Geyer M, Couillard-Despres S, Aigner R, Bogdahn U, Aigner L, Kuhn G, Winkler J (2006) Striatal deafferentation increases dopaminergic neurogenesis in the adult olfactory bulb. Exp Neurol 197:113-121. https://doi.org/10.1016/j.expneurol. 2005.08.028

75. Lennington JB, Pope S, Goodheart AE, Drozdowicz L, Daniels SB, Salamone JD, Conover JC (2011) Midbrain dopamine neurons associated with reward processing innervate the neurogenic subventricular zone. J Neurosci 31:13078-13087. https://doi.org/ 10.1523/JNEUROSCI.1197-11.2011

76. Xiong A, Wesson DW (2016) Illustrated review of the ventral striatum's olfactory tubercle. Chem Senses 41:549-555. https:// doi.org/10.1093/chemse/bjw069

77. Zhang Z, Liu Q, Wen P, Zhang J, Rao X, Zhou Z, Zhang H, He X et al (2017) Activation of the dopaminergic pathway from VTA to the medial olfactory tubercle generates odor-preference and reward. Elife 6. https://doi.org/10.7554/eLife.25423

78. Zhang Z, Zhang H, Wen P, Zhu X, Wang L, Liu Q, Wang J, He X et al (2017) Whole-brain mapping of the inputs and outputs of the medial part of the olfactory tubercle. Front Neural Circuits 11:52. https://doi.org/10.3389/fncir.2017.00052

79. McLean JH, Shipley MT, Nickell WT, Aston-Jones G, Reyher CK (1989) Chemoanatomical organization of the noradrenergic input from locus coeruleus to the olfactory bulb of the adult rat. J Comp Neurol 285:339-349. https://doi.org/10.1002/cne.902850305
80. Steinfeld R, Herb JT, Sprengel R, Schaefer AT, Fukunaga I (2015) Divergent innervation of the olfactory bulb by distinct raphe nuclei. J Comp Neurol 523:805-813. https://doi.org/10.1002/cne.23713

81. Brunert D, Tsuno Y, Rothermel M, Shipley MT, Wachowiak M (2016) Cell-type-specific modulation of sensory responses in olfactory bulb circuits by serotonergic projections from the raphe nuclei. J Neurosci 36:6820-6835. https://doi.org/10.1523/JNEUROSCI. 3667-15.2016

82. Moriizumi T, Tsukatani T, Sakashita H, Miwa T (1994) Olfactory disturbance induced by deafferentation of serotonergic fibers in the olfactory bulb. Neuroscience. 61:733-738. https://doi.org/10.1016/ 0306-4522(94)90396-4

83. Glasl L, Kloos K, Giesert F, Roethig A, Di Benedetto B, Kühn R et al (2012) Pink1-deficiency in mice impairs gait, olfaction and serotonergic innervation of the olfactory bulb. Exp Neurol 235: 214-227. https://doi.org/10.1016/j.expneurol.2012.01.002

84. Halliday GM, Blumbergs PC, Cotton RGH, Blessing WW, Geffen LB (1990) Loss of brainstem serotonin- and substance P-containing neurons in Parkinson's disease. Brain Res 510:104-107. https://doi. org/10.1016/0006-8993(90)90733-R

85. Politis M, Wu K, Loane C, Kiferle L, Molloy S, Brooks DJ, Piccini P (2010) Staging of serotonergic dysfunction in Parkinson's disease: an in vivo 11C-DASB PET study. Neurobiol Dis 40:216221. https://doi.org/10.1016/j.nbd.2010.05.028

86. Pagano G, Niccolini F, Fusar-Poli P, Politis M (2017) Serotonin transporter in Parkinson's disease: a meta-analysis of positron emission tomography studies. Ann Neurol 81:171-180. https://doi.org/ 10.1002/ana.24859

Publisher's Note Springer Nature remains neutral with regard to jurisdictional claims in published maps and institutional affiliations. 